\title{
Prediction of peptides binding to MHC class I and II alleles by temporal motif mining
}

\author{
Cem Meydan ${ }^{1}$, Hasan H Otu, ${ }^{2,3}$ Osman Uğur Sezerman ${ }^{1 *}$ \\ From The Eleventh Asia Pacific Bioinformatics Conference (APBC 2013) \\ Vancouver, Canada. 21-24 January 2013
}

\begin{abstract}
Background: MHC (Major Histocompatibility Complex) is a key player in the immune response of most vertebrates. The computational prediction of whether a given antigenic peptide will bind to a specific MHC allele is important in the development of vaccines for emerging pathogens, the creation of possibilities for controlling immune response, and for the applications of immunotherapy. One of the problems that make this computational prediction difficult is the detection of the binding core region in peptides, coupled with the presence of bulges and loops causing variations in the total sequence length. Most machine learning methods require the sequences to be of the same length to successfully discover the binding motifs, ignoring the length variance in both motif mining and prediction steps. In order to overcome this limitation, we propose the use of time-based motif mining methods that work position-independently.

Results: The prediction method was tested on a benchmark set of 28 different alleles for MHC class I and 27 different alleles for MHC class II. The obtained results are comparable to the state of the art methods for both MHC classes, surpassing the published results for some alleles. The average prediction AUC values are 0.897 for class I, and 0.858 for class 11 .

Conclusions: Temporal motif mining using partial periodic patterns can capture information about the sequences well enough to predict the binding of the peptides and is comparable to state of the art methods in the literature. Unlike neural networks or matrix based predictors, our proposed method does not depend on peptide length and can work with both short and long fragments. This advantage allows better use of the available training data and the prediction of peptides of uncommon lengths.
\end{abstract}

\section{Background}

MHC (Major Histocompatibility Complex) is a large gene family with an important role in the immune system, autoimmunity, and reproduction. MHC molecules assume roles in the presentation of peptides, including self and non-self (antigenic) on their surface to T-cells. $\mathrm{T}$-cells recognize antigenic peptides and trigger a cascade of events which leads to the destruction of pathogens and infected cells. Since MHCs have a key role in immune response, they are critical in many diseases, and can be used for controlling specific immunological processes by creating peptides to bind to specific MHC alleles. This

\footnotetext{
* Correspondence: ugur@sabanciuniv.edu

'Bioengineering Department, Sabancı University, 34956, Istanbul, Turkey Full list of author information is available at the end of the article
}

binding affinity to specific peptides may be exploited for creating peptide vaccines for emerging pathogens [1], suppressing specific alleles in organ transplants [2,3], and many other possible areas in immunotherapy.

MHC class I molecules bind short peptides, whose Nand $\mathrm{C}$-terminal ends are anchored into the pockets located at the ends of the peptide binding groove [4]. While the majority of the peptides are of length 9 , longer peptides can be accommodated by the bulging of their central portion $[5,6]$, resulting in binding peptides of length 8 to 15 [7]. Peptides binding to class II proteins are not constrained in size $[8,9]$ and can vary from 11 to 30 amino acids long [10]. The peptide binding groove in the MHC class II molecules is open at both ends, which enables binding of peptides with relatively longer length. 
Though the "core" nine residues long segment contributes the most to the recognition of the peptide, the flanking regions are also important for the specificity of the peptide to the class II allele [11,12]. MHC molecules bind peptides with high promiscuity; it is estimated that each HLA (human leukocyte antigen system) protein can bind between 1000 and 10,000 peptides for class I allotypes [13] and more than 2000 peptides for class II allotypes [14]. Thus, the large number of possible structures makes it unfeasible to find peptides that will bind to a specific allele using solely an experimental approach.

Computational methods for prediction of the binding affinity of a peptide to an MHC allele are based on three main artificial learning systems: statistical, structural, and neural methods $[13,15,16]$. The combination of these models is also common [17]. Computational approaches available for predicting MHC binding peptides from amino acid sequences include: (i) Motif-based methods such as methods that use a position weight matrix (PWM) to model a gapless multiple sequence alignment of MHC binding peptides [4-8], and a statistical approach based on Hidden Markov Models (HMMs) $[9,10]$; (ii) Machine learning methods based on Artificial Neural Networks (ANN) [6,11-13] and Support Vector Machines (SVMs) [14-17]; (iii) Semi-supervised machine learning methods $[18,19]$. Existing methods are reviewed in detail in $[18,19]$.

The formation of bulges and loops may allow peptides that are shorter or longer than 9 amino acids to bind to class I alleles. This length variance shifts the positions of amino acids in anchor locations, causing position-specific scoring matrices or other position-dependent methods to fail. Most existing methods enforce a length constraint of 9 peptides for class I prediction. ANN, quantitative matrices and similar methods require the peptides to be of the same length, with appropriate peptides aligned in the same location. Peptides of different lengths are either ignored or grouped into separate datasets by their length. This step may not always be feasible if the data is limited, especially for short and variable peptides.

Unlike MHC class I prediction methods, most of the MHC class II prediction methods can utilize peptides of variable length. However, the prediction strategy requires the determination of the core 9-mer region of the peptide. This core segment is assumed to be fixed-length and the possibility of longer binding core sequences is disregarded. Although peptides bind to MHC class II alleles mostly by the anchor residues, the interactions of the flanking regions may be important for specificity and therefore have to be taken into account [20].

In order to overcome these obstacles, we suggest a method using partial periodic pattern mining, which does not require the peptides to be of same length or the anchor positions to be specific. We propose a novel method for extracting the motifs on peptides with variable lengths by finding partial motifs in sequence data. Our method, called MHC-PPM, may capture aforementioned variations in peptides, without filtering or pre-processing the shorter/longer peptides or treating them as separate datasets. Additionally, the information in the flanking regions of the core 9-mers is taken into account without any information loss that may have arisen due to length constraints.

\section{Methods \\ Dataset}

We used 28 different alleles from the Immune Epitope Database (IEDB) benchmark dataset by Peters et al. [21,22] for MHC class I prediction (total of 36,829 peptides). For MHC class II, we used two benchmark sets from Wang et al., 16 alleles containing 10,017 peptides [19] (referred to as Wang2008), and 26 alleles containing 44,541 peptides [23] (referred to as Wang2010). Wang2010 contains data from several different human alleles, including HLA DR, DP and DQ. Wang2010 data also contains a similarity reduced subset (SR), where sequence similarity is minimized in order to reduce the overlap between cross-validation folds. In Peters and Wang2010 datasets, the same cross-validation folds are used for comparison to the benchmark values. 10 -fold cross-validation was used in Wang2008 dataset.

The peptides from these alleles are assigned into positive and negative classes by the $\mathrm{IC}_{50}=500 \mathrm{nM}$ cut-off. Unlike other MHC prediction methods, no filtering was made with regard to length during the motif mining and prediction steps.

\section{Motif mining \\ i-) Apriori method}

Our motif mining method is based on the apriori algorithm used in frequent association rule discovery [24]. An "itemset" is defined as a set of items or events that co-occur frequently. The Apriori algorithm uses the principle that all subsets of a frequent itemset must also be frequent. Accordingly, the algorithm has a bottom-up approach where the shorter frequent itemsets are extended to create longer candidates, which are then filtered by frequency of occurrence [24-26]. This iterative extension process continues until no frequent itemsets of a certain length can be found.

Due to the context difference, the formal statement of the problem in the Apriori algorithm [26] is slightly modified. Let $I=\{i 1, i 2, \ldots, i m\}$ be an alphabet of items called events (amino acids in our case). Let $D$ be a set of sequences, where each sequence $S$ is an ordered set of items such that $S \subseteq I$. A sequence $S$ contains itemset $X$, an ordered set of some items in $I$, if $X \subseteq S$. A rule is of the form, where $X \subset I, Y \subset I$. With temporal information, $\{X \rightarrow Y\}$ also implies that the events in $X$ occur before $Y$ in a sequence $S$ containing the rule. 
The ratio of the sequences containing the association rule to all of the sequences is called the support of the rule. The ratio of the sequences containing a new rule created by the combination of two rules to the sequences containing the previous rule is called the confidence. That is,

$$
\operatorname{Conf}(X \rightarrow Y)=\frac{\operatorname{Support}(X \cup Y)}{\operatorname{Support}(X)}
$$

Our motif mining method (MHC-PPM) is similar to temporal event mining in time-related databases [27]. In general, the partial periodic pattern mining algorithms for time series data will attempt to find frequently cooccurring events, or causality relationships between them. These methods try to capture the patterns which occur in an order which is not necessarily a consecutive one. In the domain of protein motifs, the amino acids become the "events" and the causality/future prediction aspects become the motifs that are sought [28].

In the proposed approach, each sequence is taken as a separate time series, with many parallel events occurring at the same time, with each event related only to the sequence upon which it is found. In these time series, if an event happens frequently after another one within a given time window, this frequent occurrenceis considered an episode of events, a motif. To exploit the apriori principle for performance, the motifs begin from length 1. A longer motif including a specific amino acid will have support less than or equal to the support of that amino acid. Hence, if an amino acid is infrequent, any motif that includes that amino acid will also be infrequent. Thus, iteratively $\mathrm{L}_{\mathrm{N}}$ (frequent itemset of size $\mathrm{N}$ ) is created from filtering of $\mathrm{C}_{\mathrm{N}}$, candidate itemset of size $\mathrm{N}$ by $C_{N}=L_{N-1} \rightarrow L_{1}$.

First $\mathrm{L}_{1}$, the frequent itemsets of size 1 (i.e. amino acids) are found. The first step is straightforward: only the amino acids within the sequences are counted, and if an amino acid's frequency (support of the rule) is below the given threshold, the amino acid is filtered out.

Then the candidate set of size $2, C_{2}$ is created from the amino acids by $\mathrm{L}_{1} \rightarrow \mathrm{L}_{1}$, that is, the combination of any two frequent itemsets of size 1 . For example, if all of the 20 amino acids were frequent, we would have 400 candidate rules at $\mathrm{C}_{2}$ for the given parameters. Those candidate rules would then be filtered according to the preset minimum support values, yielding $\mathrm{L}_{2}$. Only a handful of those 400 rules would be frequent in the data. An example rule of size 2 would be $\{L \rightarrow V\}$, which represents Leucine followed by Valine in a window specified by parameters. The support of this candidate rule will be the ratio of occurrence of $\mathrm{L} \rightarrow \mathrm{V}$ to all of the sequences, and the confidence of the rule would be the ratio of occurrence of $\mathrm{L} \rightarrow \mathrm{V}$ to all of the sequences that contain " $\mathrm{L}$ " at some point. In other words, confidence would be the conditional probability of seeing Valine in the window, given that we observed a Leucine.

To account for the position variations in the alleles, a specific window should be defined. If an amino acid $\mathrm{X}$ is followed by $\mathrm{Y}$ after at least $\operatorname{MinS}$ and at most MaxS positions, then the rule $\{\mathrm{X} \rightarrow \mathrm{Y}\}$ is present in that sequence. If these amino acids co-occur within this window by this specific order at least minimum support times, then it is considered frequent.

In the motif mining context, the frequent rules are not simply association rules as in a shopping basket analysis; items also have a temporal value, which is used for relations such as "before" and "after" ("simultaneously" is not used in protein motifs since at each time point, that is a specific position in the sequence, only one amino acid can occur). The episode $\mathrm{A} \rightarrow \mathrm{B}$ then becomes, "whenever the events in the rule $\mathrm{A}$ occur in a given sequence, event $\mathrm{B}$ is likely to occur within $n$ to $m$ positions after $\mathrm{A}$, with $\mathrm{P}(\mathrm{A} \rightarrow \mathrm{B})$ as $p$ (support) and $\mathrm{P}(\mathrm{B} \mid$ A) as $c$ (confidence)".

There are two parameters, the slack length $(s)$, which is the length after an event within which we do not look for a rule, and the window size $(w)$, in which the consequent event may occur. Thus, $\operatorname{MinS}=s$ and $\operatorname{Max} S=s+$ $w-1$, and the rule is given as $\mathrm{A} \rightarrow \mathrm{B}(\mathrm{p}, \mathrm{c})$ for parameters $(\mathrm{s}, \mathrm{w})$. An example motif mining step is given in Figure 1 and Additional File 1 the pseudocode of the algorithm is given in Figure 2.

In our simulations, we used a window size of 1 to 3 and slack length of -8 to 8 , producing different rulesets. Negative slack values are taken by reversing the input sequences and applying the algorithm with the absolute value of the slack.

For $s=1$ and $s=-1$, the rules that consist of consecutive/nearby amino acids were mined whereas for the larger values of $s$, the motifs consisting of amino acids at separate ends of the peptide were found. Since the anchor positions of MHC motifs may be different, different slack lengths are needed to mine them all.

\section{ii-) Position dependent 1-rules}

With the addition of position information, single amino acids can be employed as rules for anchors. When mining 1-rules, the position information is kept along with the window size. Thus, an example rule with window size of 2 may be $\{\mathrm{L}$, between positions 3-4 (support: $\mathrm{p}$, confidence: c)\}. This rule will be counted as present in a peptide which includes a Leucine between the positions 3 and 4 .

\section{iii-) Recursive rule mining on training set}

In the rule mining process, the rules are mined for different slack lengths and finally 1-rules are added to the collection of rules. Following the rule mining process, all of the peptides in the training set are scored by the rules according to the Support-based prediction described below. After scoring every peptide in the training data, 
a-)

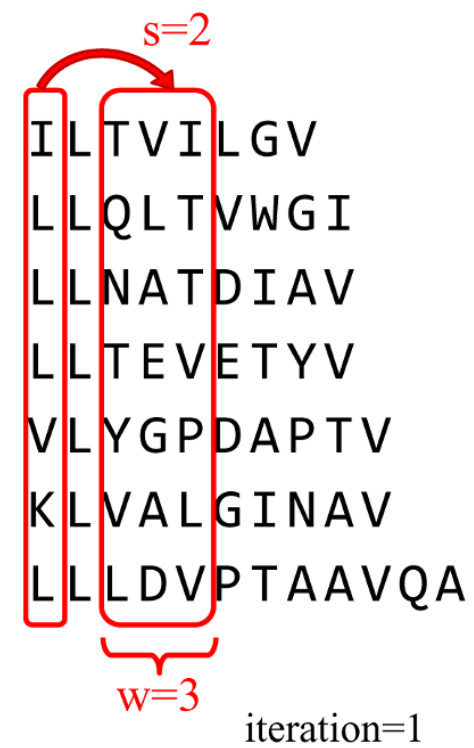

b-)

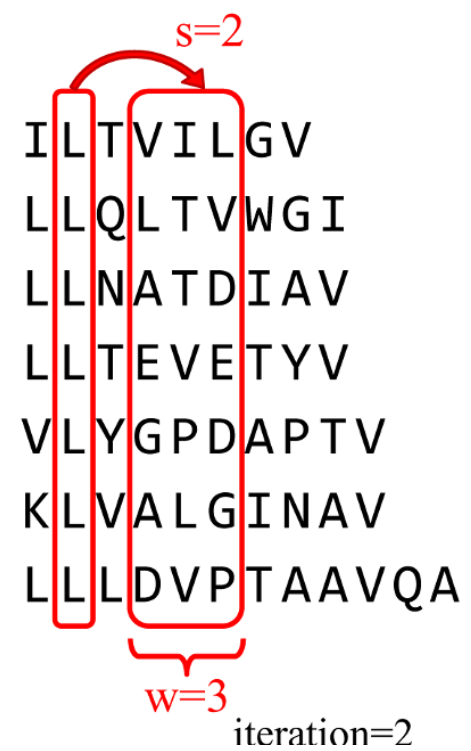

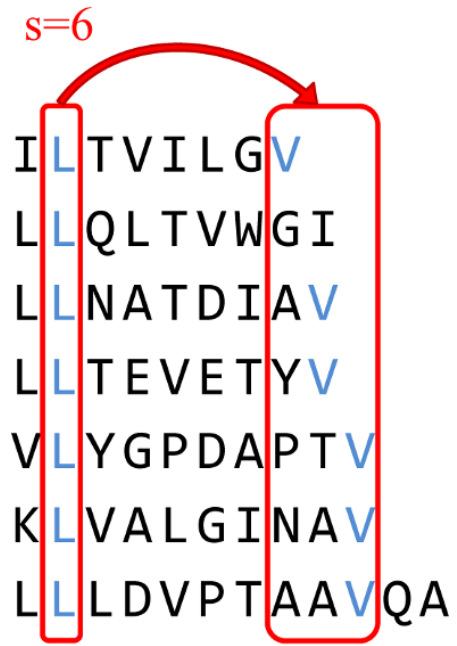

Figure 1 An example of the temporal rule mining process. a-) Schematic representation of the sliding windows approach on a sample set of sequences binding to MHC class I allele HLA $A^{*} 0201$. The windows are shifted with the given $s$ and w values until all sequences are covered. The resulting rules are then filtered by their support. This process is repeated for all $s$ values from -8 to -1 and 1 to 8 . $\mathbf{b}$-) Representation of the $L \rightarrow V$ rule captured with the parameters $s=6$ and $w=3$. For HLA $A^{*} 0201$, Leucine in $2^{\text {nd }}$ position and Valine around $9^{\text {th }}$ position is a wellknown binding motif [40]. Although this motif is present in 6 of the 7 sequences (support of 0.86), it is unlikely to be captured by position specific methods due to length variance and positional shifts.

any peptide scoring below a predefined threshold is separated. Those separated peptides that are not sufficiently explained by the motifs are fed into the motif mining recursively.

This process can be thought of as mining rules for different clusters of sequences; the first iteration will try to capture the motifs for the cluster with the most sequences. After that, sequences that scored poorly will be used in motif mining again in the second iteration, and since the data is only a subset of the previous iteration, the limit for reaching minimum support will be lower. This process is repeated until the number of peptides that score lower than the threshold is below a predefined limit, until no more improvement can be gained by dividing the dataset or until a hard limit on iteration is reached. The supports for the newly mined rules are updated to reflect the support in all of the data, not the subset. An overall view of the recursive rule mining steps are given in Figure 3.

The recursive rule mining has advantages compared to setting the minimum support and confidence threshold to lower values and mining the rules in one pass. If the rules are mined in one pass with a very low support threshold, a greater number of rules will be found. Unless those rules are significant, the signal-to-noise ratio will decrease. By using a greater initial support value and progressively decreasing it on only a subset of data, the number of possible rules is reduced; if the first pass can capture motifs that are present in $70 \%$ of all sequences, we will only mine rules for explaining the remaining $30 \%$, not the entire dataset. Hence, we end up with a lower number of more significant rules that explain the majority of the data.

\section{Prediction}

Before prediction, rules from both the binding and nonbinding sequences are mined separately. During classification of an unknown peptide, the peptide is scored independently by both the binding and non-binding rules. The simplest classification method is the direct comparison of the scores for binding/non-binding rules. To calculate the scores, the support values of the rules that occur in the given peptide are summed for both classes. The peptide is predicted to belong to the class with a higher score. This naïve approach is called Support-based prediction and only used during the recursive rule mining step.

The presence of one significant negative motif can turn an otherwise strongly binding peptide to a non-binding 


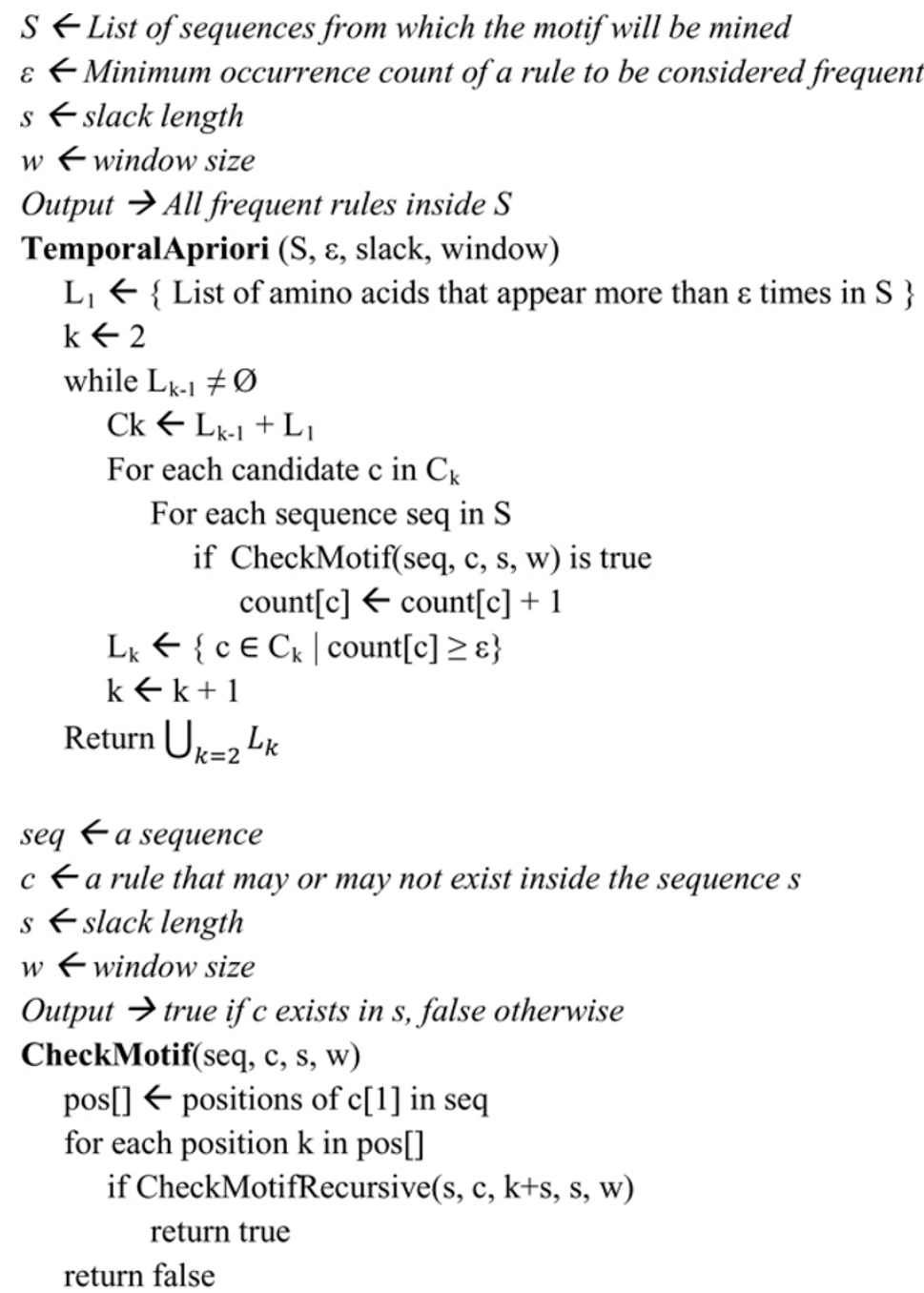

\section{CheckMotifRecursive(seq, c, i, s, w)}

$\mathrm{e} \leftarrow \mathrm{c}[\mathrm{i}]$

pos[] $\leftarrow$ positions of e in seq

for each position $\mathrm{k}$ in pos[]

if $\mathrm{e}$ is the last event in $\mathrm{c}$

return true

else if $\mathrm{k}+\mathrm{s} \leq$ length(seq)

ss $\leftarrow$ substring of seq starting from $\mathrm{k}+\mathrm{s}$ to the end

if CheckMotifRecursive(ss, c, i+1, s, w) is true

return true

return false

Figure 2 Pseudocode of the temporal apriori motif mining algorithm.

one. For example, in the allele $\mathrm{H}-2 \mathrm{Kd}$, charged or bulky amino acids inhibit binding when they are present at the $5^{\text {th }}$ position, even though the binding motif may also be present [29]. In a naïve prediction method, if the binding motifs are strong enough, the large number of binder rules will overpower the single negative motif, causing a false positive. Consequently, there is need for a way to predict these enhancing/inhibiting effects of the rules. 


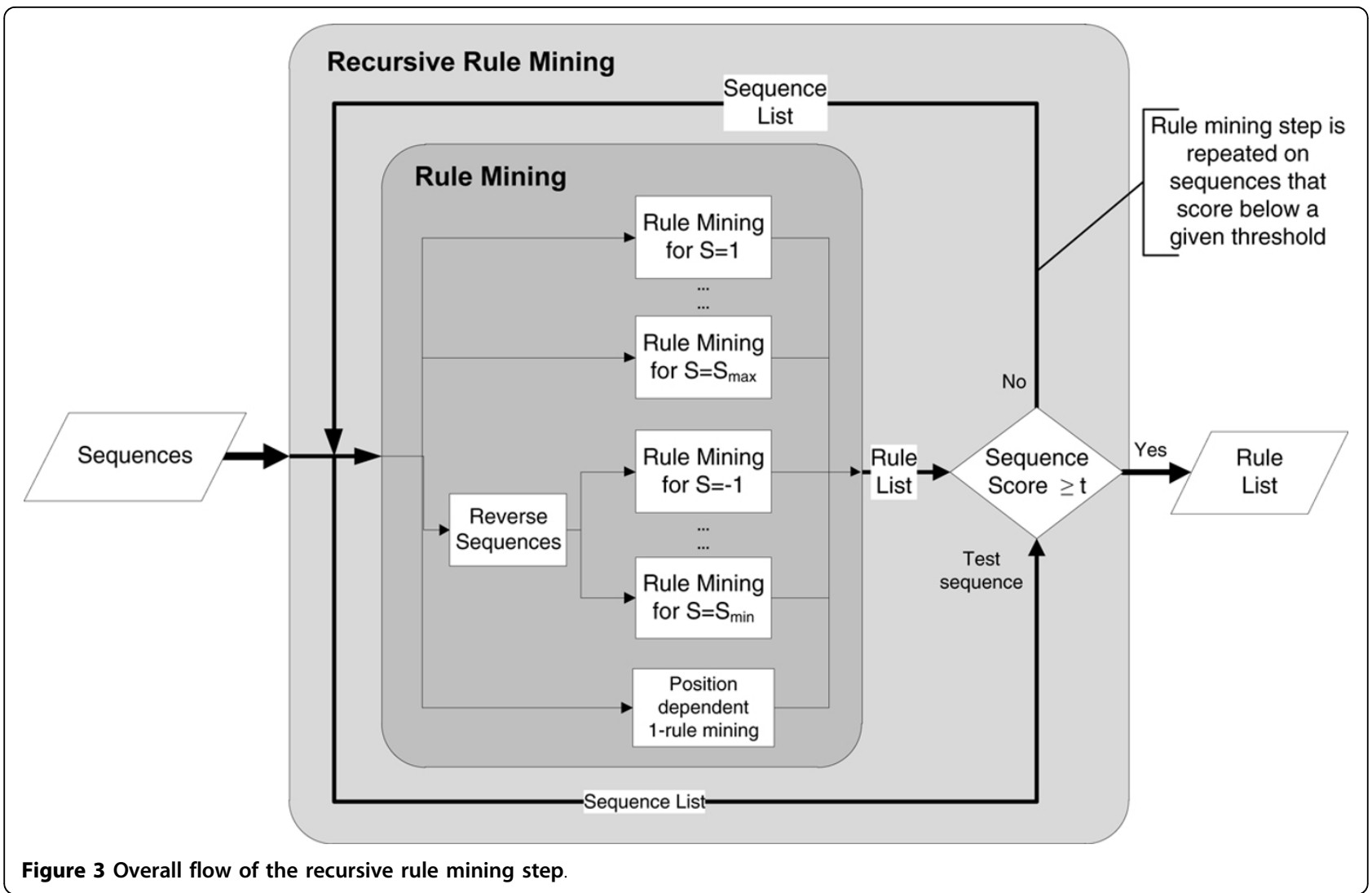

Non-linear classification methods that intrinsically find the discriminant function on the feature space would fare better in such data.

For SVR-based prediction, motif mining is employed on the training data as described above. Using the motifs, a dataset is built by creating a binary matrix, where each row is a peptide and each column (feature) represents a motif. A cell has the value 1 if the peptide corresponding to that row includes the motif, otherwise 0 . As additional columns, the sums of support and confidence scores for both the positive and negative classes are given. This data matrix is built for both the training and the test sets. Then, an SVR is trained on the training set, and the binding affinities of the peptides in the test set are predicted by the support vectors. The resulting binding affinity values can be converted into a binary class using an $\mathrm{IC}_{50}$ threshold where a binary class is required, such as feature selection methods or AUC calculation.

Since the training set is used in all of the rule mining, SVR training and parameter optimization steps, the prediction of the test set does not include any bias and represents the actual predictive performance of MHC-PPM.

The overall view of the prediction workflow can be seen in Figure 4.

\section{Results and conclusions MHC class I}

The prediction results of the proposed method are given in Table 1 along with the benchmark results for comparison [22]. The given values represent the area under the ROC curve (AUC) for the 5 -fold cross validation using the same fold splits in the benchmark set.

Note that for some alleles (given in the top part of Table 1) the AUC values between the methods are not directly comparable because filtering of the data differs based on the prediction method in use. ANN [30] use only the 9-mers, the peptides of other lengths are filtered out. SMM uses 9-mers and 10-mers, but trained and tested independently (i.e. 9-mers belonging to an allele and 10-mers belonging to the same allele are taken as separate sets and are fed to different predictors). As stated, our method uses peptides of all lengths in the same classifier, without any filtering or separation. For the comparison in Table 1 the weighted average of AUC values using the 9 -mer and 10 -mer peptide counts are given for SMM [31] and ARB [32]. The results are directly comparable for alleles with only 9-mers.

Although superior in 9-mers, the main limitation of ANN is the need for the peptides to be of fixed length. The same constraint is also present in SMM and is 


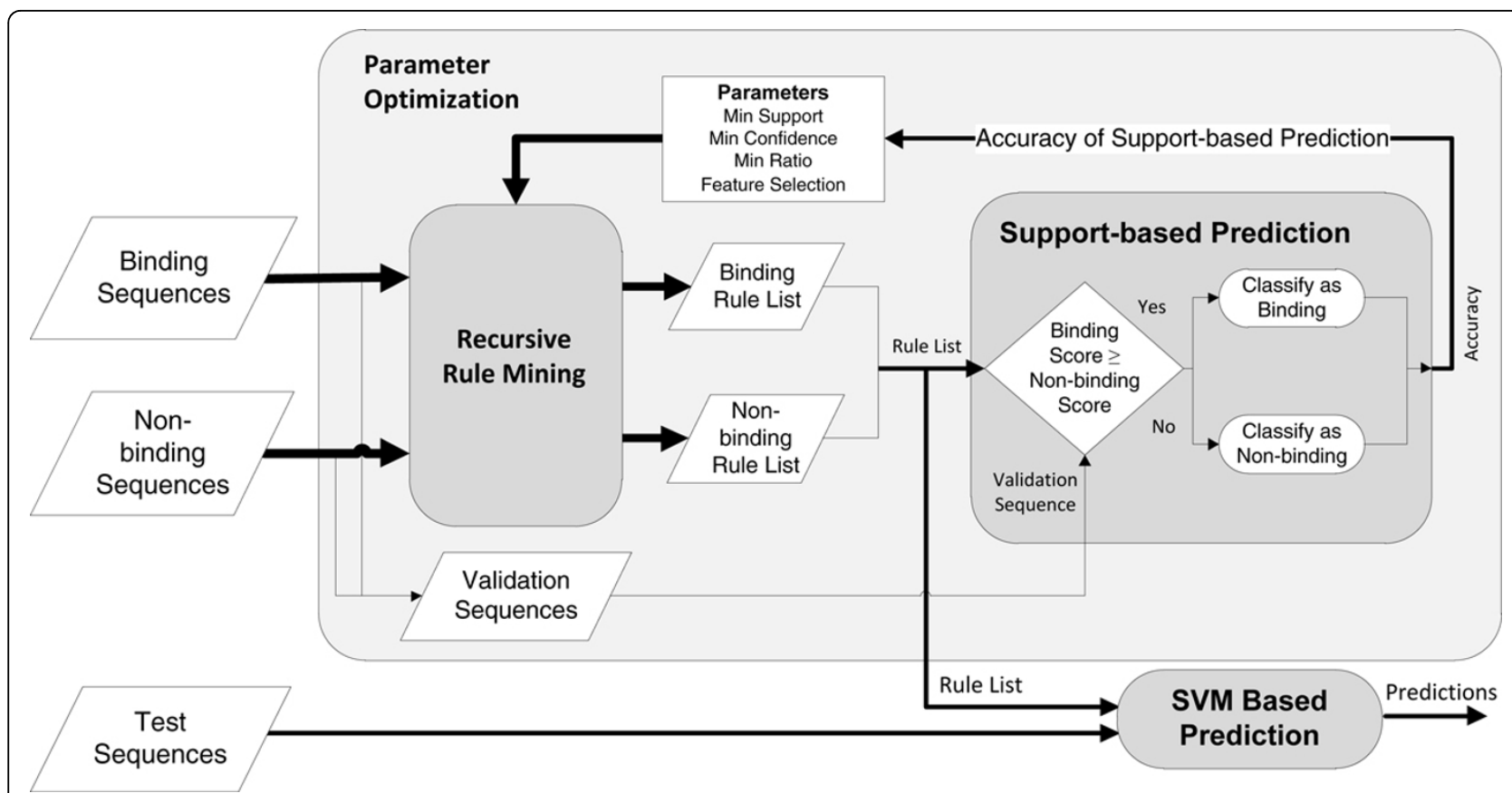

Figure 4 Overview of the experimentation process.

overcome by using separate datasets for 9-mers and 10mers. The main advantage of MHC-PPM is giving comparable and superior results to other methods without enforcing any constraints on peptide length. This flexible approach allows the use of information from all of the available data. Peptides that do not have enough representation in the dataset to train a separate classifier (e.g. 8 or 11 amino acids long) can still be predicted using the data from the 9 and 10-mers.

\section{MHC class II}

Results for Wang2008 [19] and Wang2010 [23] datasets are given in Table 2 and Table 3 respectively. Each method in the Table 3 has results for both all of the dataset (ALL) and a similarity-reduced version of the dataset (SR), used to decrease the sequence similarity between data folds.

In case of class II peptides, MHC-PPM is the top performer by the average score in the Wang2008 dataset benchmark results. However, as can be seen in the Wang2010 dataset, NN-align [33] outperforms all other methods when included in the comparison. Nonetheless, even though MHC-PPM is designed only to find position independent rules, and there are no external steps for core region detection (or any information about the core region length), it still performs exceptionally well with an average AUC value of 0.858, slightly above SMM-align [31] (AUC of 0.849) and only $<0.03$ lower than $\mathrm{NN}$-align (AUC of 0.882).

Unlike what has been observed in class I molecules, class II molecules are believed to bind only to the core 9-mer region of a peptide. Although the core region occupies the peptide binding groove, the non-bound $\mathrm{N}$ and $\mathrm{C}$-terminus residues that lie outside the MHC anchor residues, called peptide flanking residues (PFRs), have been shown to affect the binding affinity and stability $[11,34]$. NN-align and SMM-align use the length and composition of the peptide flanking residues in addition to the peptide binding core sequence. However, to keep the same length of the input throughout the data, the flanking residues are encoded in a summarized form, decreasing the information content. Due to nature of our algorithm, the differences in affinity due to the PFRs can be captured without losing any information. To test that hypothesis, we used experimental affinity values of 9 sequences which have the same core sequence and differ only in the flanking regions [11] and tried to predict the binding affinity values from the sequence (Table 4). Although available data is limited, MHC-PPM has the lowest root mean squared error (RMSE). MHC-PPM also significantly outperforms ARB, SMM-align and NN-align in correlation of the predictions with the actual affinity values.

\section{Discussions}

In this study we present a position independent motif mining method representing amino acid sequences as time series data to predict peptides binding to MHC class I and class II proteins.

In class I MHC-peptide complexes, peptides have been observed to bulge out of the binding groove $[5,6]$, shifting 
Table 1 Results of MHC-PPM in class I predictions in Peters dataset [22].

\begin{tabular}{|c|c|c|c|c|c|c|c|}
\hline & \multirow[t]{2}{*}{ Allele } & \multicolumn{2}{|c|}{ \# Peptides } & \multirow[t]{2}{*}{ ANN } & \multirow[t]{2}{*}{ ARB } & \multirow[t]{2}{*}{ SMM } & \multirow[t]{2}{*}{ MHC-PPM } \\
\hline & & 9 & 10 & & & & \\
\hline \multirow[t]{14}{*}{ 9-mers + 10-mers } & HLA-A*0201 & 3089 & 1316 & - & 0.919 & 0.939 & 0.931 \\
\hline & HLA-A*0202 & 1447 & 1056 & - & 0.851 & $\underline{0.879}$ & 0.871 \\
\hline & HLA-A*0203 & 1443 & 1055 & - & 0.838 & 0.878 & $\underline{0.882}$ \\
\hline & HLA-A*0301 & 2094 & 1082 & - & 0.883 & $\underline{0.915}$ & 0.911 \\
\hline & HLA-A*0206 & 1437 & 1054 & - & 0.849 & 0.890 & 0.885 \\
\hline & HLA-A*1101 & 1985 & 1093 & - & 0.897 & 0.932 & 0.937 \\
\hline & HLA-A*2402 & 197 & 78 & - & 0.722 & 0.809 & 0.833 \\
\hline & HLA-A*3101 & 1869 & 1057 & - & 0.881 & $\underline{0.903}$ & 0.878 \\
\hline & HLA-A*3301 & 1140 & 1055 & - & 0.866 & $\underline{0.888}$ & 0.863 \\
\hline & HLA-A*6801 & 1141 & 1055 & - & 0.827 & $\underline{0.874}$ & 0.864 \\
\hline & HLA-B*0702 & 1262 & 205 & - & 0.925 & 0.952 & $\underline{0.954}$ \\
\hline & HLA-B*3501 & 736 & 177 & - & 0.833 & $\underline{0.886}$ & 0.866 \\
\hline & HLA-B*5101 & 244 & 177 & - & 0.782 & 0.875 & $\underline{0.886}$ \\
\hline & HLA-B*5301 & 254 & 177 & - & 0.758 & $\underline{0.854}$ & 0.847 \\
\hline \multirow[t]{17}{*}{ 9-mers } & HLA-A*0101 & 1157 & - & 0.982 & 0.964 & 0.980 & 0.963 \\
\hline & HLA-A*2601 & 672 & - & $\overline{0.956}$ & 0.907 & 0.931 & 0.901 \\
\hline & HLA-A*2902 & 160 & - & 0.935 & 0.755 & 0.911 & 0.907 \\
\hline & HLA-A*6802 & 1434 & - & $\underline{0.899}$ & 0.865 & 0.898 & 0.867 \\
\hline & HLA-B*0801 & 708 & - & $\underline{0.955}$ & 0.936 & 0.943 & 0.926 \\
\hline & HLA-B*1501 & 978 & - & 0.941 & 0.900 & $\underline{0.952}$ & 0.922 \\
\hline & HLA-B*1801 & 118 & - & 0.838 & 0.573 & 0.853 & $\underline{0.906}$ \\
\hline & HLA-B*2705 & 969 & - & 0.938 & 0.915 & $\underline{0.940}$ & 0.938 \\
\hline & HLA-B*4002 & 118 & - & 0.754 & 0.541 & 0.842 & 0.891 \\
\hline & HLA-B*4402 & 119 & - & 0.778 & 0.533 & 0.740 & 0.891 \\
\hline & HLA-B*4403 & 119 & - & 0.763 & 0.461 & 0.770 & 0.847 \\
\hline & HLA-B*5401 & 255 & - & 0.903 & 0.847 & 0.921 & 0.883 \\
\hline & HLA-B*5701 & 59 & - & 0.826 & 0.428 & 0.871 & 0.929 \\
\hline & HLA-B*5801 & 988 & - & 0.961 & 0.889 & 0.964 & 0.944 \\
\hline & Average (All) & & & 0.888 & 0.798 & 0.893 & 0.897 \\
\hline & Average (9mers) & & & 0.888 & 0.751 & 0.894 & 0.908 \\
\hline & Weighted Avg & & & $\underline{0.932}$ & 0.872 & 0.910 & $\overline{0.901}$ \\
\hline
\end{tabular}

The best-performing method for each allele is underlined. The given AUC values for ARB and SMM are the weighted averages of the AUC values for 9-mers and 10 -mers based on the given peptide counts for a specific allele. The alleles in the bottom part of the table were only trained \& tested in 9-mers and are directly comparable.

the peptide side chains in the binding pockets. The main shortcoming of the existing prediction methods is their dependence on fixed length motifs, even though peptides of various lengths are known to bind to class I molecules [7]. Although a separate predictor can be created by applying the same method on a dataset of peptides of a different length, there is usually not enough available data for uncommon sequence lengths. There have been methods that use random sampling of insertions and deletions to fit the peptide into the 9-length window for prediction [35], however the fixed length limitation still present in the core.

For MHC class I predictions, MHC-PPM has been shown to slightly outperform other methods on the average. However, all methods have very close scores and perform equally well. Our main advantage is the ability to use peptides of any length during both training and prediction phases. While the curated benchmark dataset contains only 9-mers and 10-mers for the given alleles, we expect MHC-PPM to fare better in a more diverse dataset.

Commonly used prediction servers give the consensus prediction of different algorithms. The addition of our predictions into a consensus-decision step with other stateof-the-art algorithms will almost certainly benefit the end-users; the overall accuracy for the 9-mers will increase, and longer peptides that would have been previously ignored (or treated as 9-mers) will also be evaluated.

On MHC class II molecules, MHC-PPM was the top performing one in Wang2008 dataset by average AUC and just below NN-align in Wang2010 dataset. Even though NN-align outperforms all other methods including ours, the difference in performance values are not as 
Table 2 Results of MHC-PPM in class II predictions in Wang2008 dataset [19].

\begin{tabular}{|c|c|c|c|c|c|c|c|}
\hline Allele & $\#$ & RANKPEP & ARB & PROPRED & SMM-align & MHCMIR & MHC-PPM \\
\hline HLA-DRB $1 * 0101$ & 3882 & 0.700 & 0.760 & 0.740 & 0.770 & 0.810 & $\underline{0.878}$ \\
\hline HLA-DRB $1 * 0301$ & 502 & 0.670 & 0.660 & 0.650 & 0.690 & 0.640 & $\underline{0.712}$ \\
\hline HLA-DRB ${ }^{*} 0401$ & 512 & 0.630 & 0.670 & 0.690 & 0.680 & $\underline{0.730}$ & 0.666 \\
\hline HLA-DRB ${ }^{*} 0404$ & 449 & 0.660 & 0.720 & 0.790 & 0.750 & 0.730 & $\underline{0.792}$ \\
\hline HLA-DRB $1 * 0405$ & 457 & 0.620 & 0.670 & $\underline{0.750}$ & 0.690 & 0.730 & 0.734 \\
\hline HLA-DRB $1 * 0701$ & 505 & 0.580 & 0.690 & 0.780 & 0.780 & 0.830 & $\underline{0.893}$ \\
\hline HLA-DRB $1 * 0802$ & 245 & - & 0.740 & 0.770 & 0.750 & 0.740 & $\underline{0.827}$ \\
\hline HLA-DRB $1 * 0901$ & 412 & 0.610 & 0.620 & - & 0.660 & 0.620 & $\underline{0.666}$ \\
\hline HLA-DRB1*1101 & 520 & 0.700 & 0.730 & 0.800 & 0.810 & 0.810 & 0.817 \\
\hline HLA-DRB1*1302 & 289 & 0.520 & $\underline{0.790}$ & 0.580 & 0.690 & 0.720 & 0.679 \\
\hline HLA-DRB1*1501 & 520 & 0.620 & 0.700 & 0.720 & 0.740 & 0.730 & $\underline{0.759}$ \\
\hline HLA-DRB3*0101 & 420 & - & 0.590 & - & 0.680 & - & 0.712 \\
\hline HLA-DRB4*0101 & 245 & 0.650 & 0.740 & - & 0.710 & 0.760 & $\underline{0.829}$ \\
\hline HLA-DRB5*0101 & 520 & 0.730 & 0.700 & 0.790 & 0.750 & 0.710 & $\underline{0.845}$ \\
\hline $\mathrm{H}-2 \mathrm{IAb}$ & 500 & 0.740 & $\underline{0.800}$ & - & 0.750 & 0.690 & 0.786 \\
\hline $\mathrm{H}-2 \mathrm{IEd}$ & 39 & 0.830 & - & - & - & - & $\underline{0.867}$ \\
\hline Average & & 0.661 & 0.705 & 0.733 & 0.727 & 0.732 & $\underline{0.779}$ \\
\hline Weighted Avg & & 0.671 & 0.722 & 0.738 & 0.743 & 0.760 & 0.780 \\
\hline
\end{tabular}

The (\#) column gives the total number of peptides for the given allele. The best-performing method for each allele is underlined.

Table 3 Results of MHC-PPM in MHC class II predictions in Wang2010 dataset [23].

\begin{tabular}{|c|c|c|c|c|c|c|c|c|c|c|}
\hline \multirow[t]{2}{*}{ Allele } & \multicolumn{2}{|c|}{ \# Peptides } & \multicolumn{2}{|c|}{ ARB } & \multicolumn{2}{|c|}{ SMM-align } & \multicolumn{2}{|c|}{$\mathrm{NN}$-align } & \multicolumn{2}{|c|}{ MHC-PPM } \\
\hline & ALL & SR & ALL & SR & ALL & SR & ALL & SR & ALL & SR \\
\hline HLA-DPA $1 * 0103-D P B 1 * 0201$ & 1404 & 603 & 0.823 & 0.745 & 0.921 & 0.767 & 0.943 & $\underline{0.793}$ & 0.931 & 0.772 \\
\hline HLA-DPA1*01-DPB1*0401 & 1337 & 540 & 0.847 & 0.746 & 0.930 & 0.767 & 0.947 & $\underline{0.802}$ & 0.935 & 0.751 \\
\hline HLA-DPA1*0201-DPB1*0101 & 1399 & 604 & 0.824 & 0.743 & 0.909 & 0.786 & 0.944 & $\underline{0.818}$ & 0.938 & 0.806 \\
\hline HLA-DPA $1 * 0201-D P B 1 * 0501$ & 1410 & 586 & 0.859 & 0.709 & 0.923 & 0.728 & 0.956 & $\underline{0.787}$ & 0.948 & 0.773 \\
\hline HLA-DPA $1 * 0301-D P B 1 * 0402$ & 1407 & 602 & 0.821 & 0.771 & 0.932 & 0.818 & 0.949 & $\underline{0.828}$ & 0.935 & 0.815 \\
\hline HLA-DQA1*0101-DQB1*0501 & 1739 & 584 & 0.871 & 0.741 & 0.930 & 0.783 & 0.945 & 0.805 & 0.949 & $\underline{0.754}$ \\
\hline HLA-DQA 1*0102-DQB1*0602 & 1629 & 593 & 0.777 & 0.708 & 0.838 & 0.734 & 0.880 & $\underline{0.762}$ & 0.842 & 0.730 \\
\hline HLA-DQA $1 * 0301-D Q B 1 * 0302$ & 1719 & 596 & 0.748 & 0.637 & 0.807 & 0.663 & 0.851 & $\overline{0.693}$ & 0.845 & $\underline{0.709}$ \\
\hline HLA-DQA 1*0401-DQB1*0402 & 1701 & 585 & 0.845 & 0.643 & 0.896 & 0.761 & 0.922 & 0.742 & 0.920 & $\underline{0.778}$ \\
\hline HLA-DQA 1*0501-DQB1*0201 & 1658 & 589 & 0.855 & 0.700 & 0.901 & 0.736 & 0.932 & $\underline{0.777}$ & 0.919 & 0.766 \\
\hline HLA-DQA1*0501-DQB1*0301 & 1689 & 602 & 0.844 & 0.756 & 0.910 & 0.801 & 0.927 & $\underline{0.811}$ & 0.915 & 0.771 \\
\hline HLA-DRB1*0101 & 6427 & 3504 & 0.770 & 0.710 & 0.798 & 0.756 & 0.843 & $\underline{0.763}$ & 0.821 & 0.758 \\
\hline HLA-DRB1*0301 & 1715 & 1136 & 0.753 & 0.728 & 0.852 & 0.808 & 0.887 & $\underline{0.829}$ & 0.828 & 0.747 \\
\hline HLA-DRB1*0401 & 1769 & 1221 & 0.731 & 0.668 & 0.781 & 0.721 & 0.813 & $\underline{0.734}$ & 0.763 & 0.711 \\
\hline HLA-DRB1*0404 & 577 & 474 & 0.707 & 0.681 & 0.816 & 0.789 & 0.823 & 0.803 & 0.885 & $\underline{0.717}$ \\
\hline HLA-DRB1*0405 & 1582 & 1049 & 0.771 & 0.716 & 0.822 & 0.767 & 0.870 & $\underline{0.794}$ & 0.831 & 0.734 \\
\hline HLA-DRB1*0701 & 1745 & 1175 & 0.767 & 0.736 & 0.834 & 0.796 & 0.869 & $\underline{0.811}$ & 0.846 & 0.804 \\
\hline HLA-DRB1*0802 & 1520 & 1017 & 0.702 & 0.649 & 0.741 & 0.689 & 0.796 & $\underline{0.698}$ & 0.752 & 0.687 \\
\hline HLA-DRB1*0901 & 1520 & 1042 & 0.747 & 0.654 & 0.765 & 0.696 & 0.810 & $\underline{0.713}$ & 0.762 & 0.671 \\
\hline HLA-DRB1*1101 & 1794 & 1204 & 0.800 & 0.777 & 0.864 & 0.829 & 0.900 & $\underline{0.847}$ & 0.858 & 0.811 \\
\hline HLA-DRB1*1302 & 1580 & 1070 & 0.727 & 0.667 & 0.797 & $\underline{0.754}$ & 0.814 & 0.732 & 0.768 & 0.717 \\
\hline HLA-DRB1*1501 & 1769 & 1171 & 0.763 & 0.696 & 0.796 & 0.741 & 0.852 & $\underline{0.756}$ & 0.813 & 0.745 \\
\hline HLA-DRB3*0101 & 1501 & 987 & 0.709 & 0.678 & 0.819 & 0.780 & 0.856 & $\underline{0.798}$ & 0.782 & 0.718 \\
\hline HLA-DRB4*0101 & 1521 & 1011 & 0.785 & 0.747 & 0.816 & 0.762 & 0.870 & $\underline{0.789}$ & 0.860 & 0.772 \\
\hline HLA-DRB5*0101 & 1769 & 1198 & 0.760 & 0.697 & 0.832 & 0.776 & 0.886 & 0.795 & 0.843 & $\underline{0.812}$ \\
\hline $\mathrm{H}-2-\mathrm{lab}$ & 660 & 546 & 0.800 & 0.775 & 0.855 & 0.830 & 0.858 & $\underline{0.847}$ & 0.824 & 0.807 \\
\hline Average & & & 0.785 & 0.711 & 0.849 & 0.763 & 0.882 & $\underline{0.782}$ & 0.858 & 0.755 \\
\hline WeightedAverage & & & 0.784 & 0.709 & 0.843 & 0.762 & 0.879 & $\underline{0.778}$ & 0.853 & 0.754 \\
\hline
\end{tabular}

Each method contains results from all of the peptides (ALL) and the similarity reduced data (SR). The best-performing method for each allele in ALL dataset is marked by bold and the best performing method in SR dataset is underlined. 
Table 4 Effect of flanking peptides on the binding affinity to HLA DRB1*1501 allele.

\begin{tabular}{|c|c|c|c|c|c|c|}
\hline Sequence & $\begin{array}{c}\text { Experimental } \\
\text { IC50(nM) }\end{array}$ & $\begin{array}{c}\text { ARB } \\
1 \mathrm{C} 50(\mathrm{nM})\end{array}$ & $\begin{array}{c}\text { NetMHCIIpan } \\
\text { IC50(nM) }\end{array}$ & $\begin{array}{c}\text { SMM_align } \\
\text { IC50(nM) }\end{array}$ & $\begin{array}{l}\text { NN_align } \\
\text { IC50(nM) }\end{array}$ & $\begin{array}{r}\text { MHC-PPM } \\
\text { IC50(nM) }\end{array}$ \\
\hline ENPWHFFKNIVTPR & 33 & 21.9 & 10 & 21 & 8 & 11 \\
\hline WHFFKNIVHAAA & 33 & 21.9 & 9.2 & 52 & 10.7 & 139 \\
\hline WHFFKNIVTAAA & 45 & 21.9 & 9.5 & 20 & 11.5 & 224 \\
\hline WHFFKNIVTKAA & 35 & 21.9 & 8.1 & 20 & 10.5 & 142 \\
\hline WHFFKNIVTAKA & 4 & 21.9 & 8.1 & 20 & 9.8 & 83 \\
\hline WHFFKNIVTAAK & 5 & 21.9 & 8.9 & 20 & 11.1 & 263 \\
\hline DAWHFFKNITVA & 326 & 82.5 & 23.6 & 25 & 23.8 & 316 \\
\hline ADWHFFKNITVA & 454 & 82.5 & 23.8 & 25 & 23.9 & 320 \\
\hline AADVHFFKNITVA & 264 & 1286.7 & 45 & 30 & 74.4 & 392 \\
\hline RMSE & & 371.90 & 190.78 & 191.84 & 187.13 & 134.35 \\
\hline Pearson's Corr. & & 0.349 & 0.728 & 0.041 & 0.540 & 0.721 \\
\hline
\end{tabular}

Experimental affinity measurements are from [11]. Predictions of other values calculated from the IEDB website[21]. MHC-PPM has the lowest root mean squared error (RMSE) and has a correlation score approximately equal to the top performing method.

drastic. Due to the fixed size core region, length independence is not much of an issue during score calculation. On the other hand, the position independence allows the inherent detection of the core region and allows better representation of the peptide flanking residues. During the prediction of the effects of PFRs on binding affinity (Table 4), MHC-PPM resulted in the highest agreement with the experimental data, though more data is required for conclusive results.

On the subject of peptides binding to MHC class II molecules, the current view is that the peptides lie on a shallow groove with multiple contacts along the entire length of the peptide binding groove $[9,36]$. This view does not address the possibility of peptides bulging out from the groove. There have been studies that proposed examples of peptide bulging (i.e. core binding region longer than 9 amino acids) in class II molecules for several alleles [9,37-39]. Even though it is not known whether this is a general phenomenon for all class II alleles, it is possible that certain alleles can anchor peptides sufficiently at their $\mathrm{N}$ - and C-terminals to allow bulges, similar to class I molecules. If that is the case, a length insensitive method is required to correctly identify such examples, since NNalign and other methods require a fixed length core sequence.

The strength of MHC-PPM is its ability to capture length independent short motifs that are in close vicinity. Because the motif mining and prediction steps are uncoupled, the method can be used for different purposes. We have shown that the rules mined from the data can be used in conjunction with support vector machines or neural networks for non-linear prediction of any label (or quantitative value) that is correlated with the sequence motifs. However, the actual output of the algorithm is a collection of human-understandable rules and those motifs can be used as templates during sequence analysis or synthesis. Other than MHC binding predictions, the MHC-PPM method can also be applied to find motifs in gapped sequences, such as TCR recognition or receptorligand prediction problems. It is straightforward to extend the method to mine multiple groups of short sequence motifs (separated by relatively long distances) which co-occur frequently. We believe this approach can help uncover previously overlooked subtle sequence motifs in any large scale data.

\section{Additional material}

Additional File 1: The candidate and frequent itemsets of all lengths for the given example sequences in Figure 1, for minimum support value of $\mathbf{0 . 4}$. Red/bold values represent rules above the support threshold. At each step a candidate set $C_{k}$ is generated by extending the last frequent itemset $L_{k-1}$, then the candidates are filtered according to the support values to generate the frequent itemset $L_{k}$. This process is repeated until no frequent itemsets of a certain size can be found. Afterwards, the resulting frequent sets of different sizes (except $L_{1}$ ) are merged together and filtered according to a given minimum confidence boundary.

\section{Authors' contributions}

CM implemented the algorithm, carried out the experiments, contributed to the design of the study and drafted the manuscript. OUS and $\mathrm{HHO}$ contributed to the design as well. CM and OUS analyzed the results. CM, OUS and $\mathrm{HHO}$ participated in the analysis and the discussion. All authors read and approved the final manuscript.

\section{Competing interests}

The authors declare that there are no competing interests

\section{Acknowledgements}

CM would like to thank TUBITAK BIDEB (The Scientific and Technological Research Council of Turkey, Department of Science Fellowships and Grant Programmes) for support. $\mathrm{HHO}$ is partially supported by a grant from The Dubai Harvard Foundation for Medical Research.

\section{Declarations}

The publication costs for this article were funded by the corresponding author. 
This article has been published as part of BMC Bioinformatics Volume 14 Supplement 2, 2013: Selected articles from the Eleventh Asia Pacific Bioinformatics Conference (APBC 2013): Bioinformatics. The full contents of the supplement are available online at http://www.biomedcentral.com/ bmcbioinformatics/supplements/14/S2.

\section{Author details}

${ }^{1}$ Bioengineering Department, Sabancı University, 34956, Istanbul, Turkey. 2Department of Medicine, BIDMC Genomics Center, Harvard Medical School, Boston, MA 02215, USA. ${ }^{3}$ Department of Bioengineering, Istanbul Bilgi University, 34060, Istanbul, Turkey.

\section{Published: 21 January 2013}

\section{References}

1. Rosenberg SA, Yang JC, Schwartzentruber DJ, Hwu P, Marincola FM, Topalian SL, Restifo NP, Dudley ME, Schwarz SL, Spiess PJ, et al: Immunologic and therapeutic evaluation of a synthetic peptide vaccine for the treatment of patients with metastatic melanoma. Nat Med 1998, 4(3):321-327

2. Zavazava N, Fandrich F, Zhu X, Freese A, Behrens D, Yoo-Ott KA: Oral feeding of an immunodominant MHC donor-derived synthetic class I peptide prolongs graft survival of heterotopic cardiac allografts in a high-responder rat strain combination. J Leukoc Biol 2000, 67(6):793-800.

3. Murphy B, Kim KS, Buelow R, Sayegh MH, Hancock WW: Synthetic MHC class I peptide prolongs cardiac survival and attenuates transplant arteriosclerosis in the Lewis->Fischer 344 model of chronic allograft rejection. Transplantation 1997, 64(1):14-19.

4. Natarajan K, Li H, Mariuzza RA, Margulies DH: MHC class I molecules, structure and function. Rev Immunogenet 1999, 1(1):32-46.

5. Guo HC, Jardetzky TS, Garrett TP, Lane WS, Strominger JL, Wiley DC: Different length peptides bind to HLA-Aw68 similarly at their ends but bulge out in the middle. Nature 1992, 360(6402):364-366.

6. Speir JA, Stevens J, Joly E, Butcher GW, Wilson IA: Two different, highly exposed, bulged structures for an unusually long peptide bound to rat MHC class I RT1-Aa. Immunity 2001, 14(1):81-92.

7. Schumacher TN, De Bruijn ML, Vernie LN, Kast WM, Melief CJ, Neefjes JJ, Ploegh HL: Peptide selection by MHC class I molecules. Nature 1991, 350(6320):703-706

8. Nelson CA, Fremont DH: Structural principles of MHC class II antigen presentation. Rev Immunogenet 1999, 1(1):47-59.

9. Yassai M, Afsari A, Garlie J, Gorski J: C-terminal anchoring of a peptide to class II MHC via the P10 residue is compatible with a peptide bulge. J Immunol 2002, 168(3):1281-1285.

10. Rammensee $H G$, Friede T, Stevanoviic S: MHC ligands and peptide motifs: first listing. Immunogenetics 1995, 41(4):178-228.

11. Godkin AJ, Smith KJ, Willis A, Tejada-Simon MV, Zhang J, Elliott T, Hill AV: Naturally processed HLA class II peptides reveal highly conserved immunogenic flanking region sequence preferences that reflect antigen processing rather than peptide-MHC interactions. J Immunol 2001, 166(11):6720-6727.

12. Jones EY, Fugger $\mathrm{L}$, Strominger $\mathrm{L}$, Siebold C: MHC class II proteins and disease: a structural perspective. Nat Rev Immunol 2006, 6(4):271-282.

13. Brusic V, Bajic VB, Petrovsky N: Computational methods for prediction of T-cell epitopes-a framework for modelling, testing, and applications. Methods 2004, 34(4):436-443.

14. Marsh SGE, Parham P, Barber LD: The HLA factsbook. San Diego: Academic Press; 2000.

15. Schalkoff RJ: Pattern recognition: statistical, structural, and neural approaches. New York: J. Wiley; 1992.

16. Firebaugh MW: Artificial intelligence: a knowledge-based approach. Boston: Boyd \& Fraser; 1988.

17. Trost B, Bickis M, Kusalik A: Strength in numbers: achieving greater accuracy in MHC-I binding prediction by combining the results from multiple prediction tools. Immunome Res 2007, 3:5.

18. Nielsen M, Lund O, Buus S, Lundegaard C: MHC class II epitope predictive algorithms. Immunology 2010, 130(3):319-328.

19. Wang P, Sidney J, Dow C, Mothe B, Sette A, Peters B: A systematic assessment of $\mathrm{MHC}$ class II peptide binding predictions and evaluation of a consensus approach. PLoS Comput Biol 2008, 4(4):e1000048.
20. Matsumura M, Fremont DH, Peterson PA, Wilson IA: Emerging principles for the recognition of peptide antigens by MHC class I molecules. Science 1992, 257(5072):927-934.

21. Peters B, Sidney J, Bourne P, Bui HH, Buus S, Doh G, Fleri W, Kronenberg M, Kubo R, Lund $O$, et al: The immune epitope database and analysis resource: from vision to blueprint. PLOS Biol 2005, 3(3):e91.

22. Peters B, Bui HH, Frankild S, Nielson M, Lundegaard C, Kostem E, Basch D, Lamberth $K$, Harndahl M, Fleri W, et al: A community resource benchmarking predictions of peptide binding to MHC-I molecules. PLOS Comput Biol 2006, 2(6):e65.

23. Wang P, Sidney J, Kim Y, Sette A, Lund O, Nielsen M, Peters B: Peptide binding predictions for HLA DR, DP and DQ molecules. BMC Bioinformatics 2010, 11:568.

24. Agrawal $R$, Imielinski T, Swami A: Mining association rules between sets of items in large databases. Proceedings of the 1993 ACM SIGMOD international conference on Management of data Washington, D.C., United States: ACM; 1993.

25. Srikant $R$, Agrawal R: Mining generalized association rules. Future Generation Computer Systems 1997, 13(2-3):161-180.

26. Agrawal R, Srikant R: Fast Algorithms for Mining Association Rules in Large Databases. Proceedings of the 20th International Conference on Very Large Data Bases Morgan Kaufmann Publishers Inc; 1994.

27. Roddick JF, Spiliopoulou M: A bibliography of temporal, spatial and spatiotemporal data mining research. SIGKDD Explor News/ 1999, 1(1):34-38.

28. Mannila $H$, Toivonen $H$, Verkamo Al: Discovery of frequent episodes in event sequences. Data Mining and Knowledge Discovery 1997, 1(3):259-289.

29. Mitaksov V, Fremont DH: Structural definition of the H-2Kd peptidebinding motif. J Biol Chem 2006, 281(15):10618-10625.

30. Nielsen M, Lundegaard C, Worning P, Lauemoller SL, Lamberth K, Buus S, Brunak S, Lund O: Reliable prediction of T-cell epitopes using neural networks with novel sequence representations. Protein Sci 2003, 12(5):1007-1017.

31. Nielsen M, Lundegaard C, Lund O: Prediction of MHC class II binding affinity using SMM-align, a novel stabilization matrix alignment method. BMC Bioinformatics 2007, 8:238.

32. Bui HH, Sidney J, Peters B, Sathiamurthy M, Sinichi A, Purton KA, Mothe BR, Chisari FV, Watkins DI, Sette A: Automated generation and evaluation of specific MHC binding predictive tools: ARB matrix applications. Immunogenetics 2005, 57(5):304-314.

33. Nielsen $\mathrm{M}$, Lund $\mathrm{O}$ : NN-align. An artificial neural network-based alignment algorithm for MHC class II peptide binding prediction. BMC Bioinformatics 2009, 10:296.

34. Rotzschke O, Falk K, Mack J, Lau JM, Jung G, Strominger JL: Conformational variants of class II MHC/peptide complexes induced by $\mathrm{N}$ - and $\mathrm{C}$ terminal extensions of minimal peptide epitopes. Proc Natl Acad Sci USA 1999, 96(13):7445-7450.

35. Lundegaard C, Lund $O$, Nielsen M: Accurate approximation method for prediction of class I MHC affinities for peptides of length 8, 10 and 11 using prediction tools trained on 9mers. Bioinformatics 2008, 24(11):1397-1398.

36. Scott CA, Peterson PA, Teyton L, Wilson IA: Crystal structures of two I-Adpeptide complexes reveal that high affinity can be achieved without large anchor residues. Immunity 1998, 8(3):319-329.

37. Fleckenstein $B$, Kalbacher $H$, Muller CP, Stoll D, Halder $T$, Jung G, Wiesmuller $\mathrm{KH}$ : New ligands binding to the human leukocyte antigen class II molecule DRB1*0101 based on the activity pattern of an undecapeptide library. Eur J Biochem 1996, 240(1):71-77.

38. Kropshofer H, Max H, Muller CA, Hesse F, Stevanovic S, Jung G, Kalbacher H: Self-peptide released from class II HLA-DR1 exhibits a hydrophobic tworesidue contact motif. J Exp Med 1992, 175(6):1799-1803.

39. Smith KJ, Pyrdol J, Gauthier L, Wiley DC, Wucherpfennig KW: Crystal structure of HLA-DR2 (DRA*0101, DRB1*1501) complexed with a peptide from human myelin basic protein. J Exp Med 1998, 188(8):1511-1520.

40. Solache A, Morgan CL, Dodi Al, Morte C, Scott I, Baboonian C, Zal B, Goldman J, Grundy JE, Madrigal JA: Identification of three HLA-A*0201restricted cytotoxic $T$ cell epitopes in the cytomegalovirus protein pp65 that are conserved between eight strains of the virus. J Immunol 1999, 163(10):5512-5518.

doi:10.1186/1471-2105-14-S2-S13

Cite this article as: Meydan et al:: Prediction of peptides binding to MHC class I and II alleles by temporal motif mining. BMC Bioinformatics 201314 (Suppl 2):S13. 